\title{
Une méthodologie pour reconstruire la mondialisation du tourisme
}

Mathis Stock et Andreea Antonescu

\section{(2) OpenEdition}

12 Journals

Édition électronique

URL : http://journals.openedition.org/tourisme/124

DOI : 10.4000/tourisme.124

ISSN : 2492-7503

Éditeur

Éditions touristiques européennes

Édition imprimée

Date de publication : 1 juin 2014

Pagination : 2-18

ISSN : 2109-5671

\section{Référence électronique}

Mathis Stock et Andreea Antonescu, « Une méthodologie pour reconstruire la mondialisation du

tourisme », Mondes du Tourisme [En ligne], 9 | 2014, mis en ligne le 15 septembre 2015, consulté le 01 mai 2019. URL : http://journals.openedition.org/tourisme/124 ; DOI : 10.4000/tourisme.124

\section{cc) (†) $\ominus$}

Mondes du tourisme est mis à disposition selon les termes de la licence Creative Commons Attribution - Pas d'Utilisation Commerciale - Pas de Modification 4.0 International. 


\section{Une méthodologie pour reconstruire la mondialisation du tourisme}

\author{
MathIs Stock \\ Professeur de géographie du tourisme \\ Institut universitaire Kurt Bösch (IUKB), Centre de \\ recherche interdisciplinaire en tourisme (Crit) \\ [mathis.stock@iukb.ch]
}

\author{
Andreea Antonescu \\ Doctorante en études du tourisme \\ Institut universitaire Kurt Bösch (IUKB), Centre de \\ recherche interdisciplinaire en tourisme (Crit) \\ [andreea.antonescu@iukb.ch]
}

Résumé. Le processus de mondialisation du tourisme se traduit par le passage d'un ensemble peu dense de lieux touristiques, localisés en quelques endroits, à un champ touristique dense, interdépendant à l'échelle mondiale. Quelle est la spatialité de cette expansion du champ touristique? Peut-on quantifier le nombre de lieux composant le système touristique à différents moments ? Afin d'y répondre, il est nécessaire de voir comment s'est construit le processus de mondialisation du tourisme, ce qui requiert des données solides. D'un point de vue géographique, on peut adopter la perspective d'analyse des lieux dont l'émergence successive comme destination, la reproduction et l'arrêt de la fonction touristique marquent le passage du temps entre 1800 et 2000. Cet article vise donc à rendre compte d'une méthodologie qui a permis de construire les données nécessaires pour répondre aux questions posées. II se focalise sur l'identification et la périodisation du nombre des lieux touristiques, avec une première cartographie de la constitution du champ touristique mondial.

Abstract. The process of globalization of tourism results in the passage of a sparse set of tourist places, located only in some parts of the world, to a dense tourist field, interrelated on a global scale. What is the spatiality of such expansion of the tourism field? Can we quantify the number of tourist places that constitute the system at different moments? To answer this question, it is necessary to see how the process of globalization of tourism is developing. From a geographical point of view, we adopt the perspective of places, whose successive emergence, reproduction and end as tourist destination is significant for the period between 1800 to 2000. This article aims at accounting on a methodology that allows for constructing the necessary data in order to answer the questions raised above. It focuses on the identification of the number and periodisation of tourist places and a first cartography of the construction of the global tourism field. 
$\mathbf{L}$

e processus de mondialisation du tourisme se traduit par le passage d'un ensemble peu dense et peu diversifié de lieux touristiques, localisés en seulement quelques endroits, à un "écoumène touristique" mondial qui coïncide avec l'étendue terrestre (Équipe Mit, 20I I). Dans la discussion du lien entre tourisme et mondialisation, l'un des éléments essentiels réside dans l'augmentation, depuis 1800 , du nombre d'endroits consacrés à la pratique touristique. Ainsi, les endroits auxquels les touristes ont accès sont devenus plus nombreux ; l'éventail spatial pour les touristes est plus différencié. D'où la nécessité de reconstruire la façon dont cette offre en termes spatiaux s'est développée.

C'est cette dimension géographique, via l'identification du nombre et de la qualité des lieux touristiques qui émergent, se reproduisent et se restructurent depuis deux cents ans, qui est au centre du présent travail. L'objectif n'est toutefois pas d'identifier le "système" des lieux touristiques à plusieurs moments de l'histoire, car cela impliquerait la mise en évidence de liens entre les lieux géographiques, ce qui est hors de notre projet cognitif. Une contribution à la reconstruction de la mondialisation du tourisme requiert des données solides qui nécessitent la prise en compte de l'émergence successive, de la reproduction et de la restructuration, voire de l'arrêt, de la fonction touristique des différents lieux géographiques. Le défi cognitif réside dans la data- tion de ces événements. Or, ces données n'existent pas; il convient de les construire.

La construction de ces données est donc l'enjeu scientifique identifié dans le présent article, auquel nous proposons une contribution méthodologique. Au moins deux problèmes se posent dans cette entreprise. Premièrement, quel est le moment d'émergence de la fonction touristique d'un lieu ? Il s'agit, d'une part, de déterminer le moment de mise en tourisme des lieux qui, à un moment donné de la période 18002000, ont été considérés comme touristiques. D'autre part, il s'agit d'identifier et de quantifier les lieux touristiques produits entre 1800 et 2000 , et donc de faire la distinction entre lieux touristiques et lieux non touristiques, ce qui est moins aisé qu'il n'y paraît. En effet, conceptuellement, il est nécessaire de définir les critères de la "touristicité" des lieux (présence ou absence de touristes, d'infrastructures touristiques, de pratiques, de discours, etc.), mais cela ne permet pas de définir des degrés de "touristicité". Empiriquement, il est difficile de construire des données qui s'y rapportent étant donné que ces éléments empiriques (différentes formes d'écriture des toponymes, mention des pratiques touristiques, du nombre d'hôtels ou de lits touristiques, des restaurants et autres attractions touristiques) ne sont pas récurrents dans les diverses sources documentaires, mais plutôt aléatoires.

Deuxièmement, comment la fonction touristique des lieux change-t- elle avec le temps ? Il s'agit d'identifier le moment d'arrêt définitif de la fonction touristique des lieux et de quantifier les lieux touristiques disparus. Comme il faut rendre compte non seulement de la production des lieux touristiques au cours du temps, mais aussi de l'éventuelle variation de leur touristicité - possibilité d'arrêt temporaire ou définitif de la fonction touristique -, il convient, en effet, pour chaque lieu touristique, de suivre l'évolution de sa touristicité au fil du temps. Cela nécessite, d'une part, d'identifier et de quantifier les lieux qui ont su maintenir leur fonction touristique tout le long de leur existence et, d'autre part, d'identifier et de quantifier les lieux à fonction touristique intermittente ou dont la fonction touristique s'arrête définitivement (dans le laps de temps considéré). Il s'agit là d'un problème conceptuel, méthodologique et empirique dans la mesure où la distinction conceptuelle entre intermittences et arrêts définitifs ne peut être effectuée de manière absolue, mais seulement pour le laps de temps considéré. En effet, plusieurs étapes sont à franchir : le choix du corpus, l'extraction des données, leur saisie et traitement, puis leur analyse et représentation cartographique.

Une fois les objectifs assignés à la recherche fixés se pose la question des outils méthodologiques pouvant être mis en œuvre pour disposer d'une base de données solide sur la touristification du monde. Dans le présent article, il s'agit de réfléchir sur les méthodologies possibles pour 
reconstruire la façon dont, à l'échelle mondiale, entre 1800 et 2000 , le nombre de lieux touristiques a augmenté et la manière dont ils ont changé de qualité, constituant ainsi un "écoumène touristique" mondial relativement plus densifié et modifiant radicalement le champ touristique mondial.

\section{Des dONNÉES DISPONIBLES POUR L'ÉCHELLE MONDIALE ?}

La mise en œuvre de cette recherche se heurte à plusieurs obstacles. Il n'existe pas, à ce jour, de données solides sur les deux cents dernières années permettant d'appréhender la touristicité des lieux géographiques définis à l'échelle locale, pour l'ensemble du monde. Tout d'abord, il n'existe pas de données quantitatives sur le nombre de lieux touristiques, sur le nombre de touristes les fréquentant, ni de données qualitatives sur le type de lieux ou le type de pratiques s'y déroulant. Les données permettant d'apprécier le changement de ces éléments au cours du temps sont absentes. La plupart des données existantes concernent l'État-nation; elles sont produites par les services étatiques ou par l'Organisation mondiale du tourisme (OMT) et ne s'intéressent pas aux référents locaux ; elles ne proposent pas d'informations quantitatives sur le tourisme au XIX ${ }^{e}$ siècle $^{(1)}$.

Plus généralement, il existe un véritable problème de données dès lors que l'intérêt cognitif vise l'échelle mondiale du tourisme. D'une part, la connaissance sur la distribution spatiale du phénomène s'appuie surtout sur les données statistiques de l'OMT, et il n'est pas possible de se contenter uniquement de cette source, peu adaptée pour une prise en compte fine de ce phénomène à l'échelle mondiale. Une seule recherche a essayé de dépasser ce cadre (Tol et al., 2007) pour tenter de mesurer l'ampleur du tourisme en combinant tourisme international et tourisme domestique au niveau national, mais également au niveau infra-étatique. Cependant, les auteurs de cette recherche utilisent une approche économétrique dans laquelle on simule les données, mais dans laquelle on ne les mesure pas, avec tous les problèmes que cela suppose. Ainsi, on ne dispose pas aujourd'hui d'une base de données comparable à celles existant sur les villes ( $c f$., par exemple, la base Géopolis de Moriconi-Ebrard (1993)), qui comprendrait, par exemple, les lieux touristiques enregistrant plus d'un million de nuitées.

\section{Construire un raisonnement au-delà du cas d'étude}

On peut construire ces données à l'échelle locale ou, comme l'a fait l'Équipe Mit (20II), à travers une recherche réalisée, pour chaque lieu étudié, en compulsant ouvrages, travaux scientifiques, données éparses. En dix ans, une base de données d'environ 3000 lieux touristiques, avec la date d'émergence de ceuxci, a ainsi été construite. Toutefois, cette base de données ne propose pas une représentativité quantitative du phénomène ; les sélections opérées ont été faites en fonction d'une représentativité spatiale (couvrir le monde entier). On ne peut donc en tirer de conclusions sur le nombre de lieux touristiques qui, par exemple, émergent entre 1800 et 1850, par rapport à la période 1900-1950. De plus, en raison de la focalisation sur la date d'émergence des lieux touristiques, aucune information sur le maintien ou l'arrêt de la fonction n'a été collectée. On ne peut pas donc en tirer de conclusions sur la reproduction des lieux touristiques, seulement sur leur émergence cumulée. On travaille donc avec l'hypothèse implicite que tous les lieux, une fois émergés, continuent à avoir une fonction touristique jusqu'à nos jours. Ces deux limites incitent à tenter une nouvelle méthodologie, fondée sur le dépouillement de guides touristiques afin d'en extraire les différents lieux mentionnés.

Certaines méthodes se servent des guides de voyage pour produire des indicateurs touristiques. Par exemple, une base de données réalisée à partir d'une analyse manuelle du contenu des guides (Dupuy et Mao, 2003 ; Knafou, 1997) a permis de réaliser des cartes de densité des sites étoilés par le Guide Vert. Tritz $(2003,2006)$ a réalisé une base de données des lieux cités (classés en "hauts lieux" et en lieux secondaires) dans les index des guides, en utilisant le Guide du routard, le Guide Vert et les Guides Bleus.

Une autre méthode, très astucieuse, est celle d'Ollivier et al (20।2) qui consiste en l'analyse, via un logiciel, de guides préalablement numé- 
risés dont on extrait le nombre d'occurrences du nom des communes d'une région. Ensuite, on compare cette liste avec une base de données des communes de cette région, obtenue auprès de l'Insee. L'indicateur ainsi établi permet de mesurer la notoriété touristique des communes. Malheureusement, nous ne pouvons pas utiliser cette méthode dans le cadre de cette recherche, car nous ne disposons pas, pour le monde entier, d'une base de données des communes (ou des lieux) que l'on pourrait comparer aux toponymes mentionnés dans les guides touristiques. En effet, la méthode utilisée par Ollivier et al. (20/2) n'est pas une méthode d'extraction des lieux, mais une méthode de comptage. On compte le nombre d'occurrences d'un toponyme dans un guide. Une fois les guides numérisés par reconnaissance de texte, le logiciel utilisé reconnaît les toponymes à compter, puisqu'il les compare à ceux qui se trouvent dans un fichier préalablement établi (les toponymes sont connues au préalable ; le logiciel sait ce qu'il doit chercher). Un tel logiciel ne serait utile dans notre démarche que s'il pouvait extraire les noms de lieux et les afficher dans un document.

En conclusion, aucune des méthodes connues n'a pris en compte le niveau mondial ni les éléments qui nous intéressent : la date de mise en tourisme d'un lieu, la date d'arrêt de sa fonction touristique ou la permanence de cette fonction, de l'émergence du lieu jusqu'à nos jours, et ce à l'échelle mondiale.

\section{Dater l'émergence des lieux touristiques : un problème méthodologique}

La datation des événements est un problème cognitif pour les historiens qui ont développé des méthodes pour répondre à la question du "quand?", notamment par la constitution d'un corpus issu d'un travail d'archive. Concernant les lieux touristiques, on peut se poser la question de savoir comment dater l' "invention" d'un lieu touristique. S'agit-il du premier touriste que l'on a pu identifier sur place? S'agit-il des premières infrastructures touristiques (hôtel, promenade, etc.) construites ? S'agit-il du classement effectué par un ministère (les stations thermales ou les sites touristiques chinois) ? La façon de répondre à ces questions a des conséquences sur le type de données à rechercher ; inversement, la définition d'un corpus implique une réponse spécifique à ces questions.

Qu'est-ce que l'on entend par datation de la mise en tourisme ? Il s'agit de l'identification du moment où un lieu est "inventé" pour le tourisme $^{(2)}$. La question se pose alors de savoir dans quelle mesure cette mise en tourisme est "documentée". Si la recherche réussit à "documenter" la datation de la mise en tourisme dans des études de cas, est-il possible alors, avec les mêmes méthodes, de déterminer la date de la mise en tourisme des lieux géographiques à l'échelle mondiale ? Pour contourner cette difficulté, nous avons redéfini notre objectif et mis en place une "interprétation métho- dologique" : nous ne cherchons pas à déterminer la date exacte de l'émergence touristique d'un lieu, mais le moment où la fonction touristique d'un lieu est confirmée par un document. Plus précisément, nous définissons le moment de mise en tourisme d'un lieu comme un événement publié dans un guide de voyage. Nous obtenons donc une date qui correspond à la première mention du lieu dans un guide de voyage. Cette date est nécessairement postérieure à la mise en tourisme effective. Nous interprétons cette mention comme la reconnaissance, par une configuration sociale constituée de touristes, d'éditeurs et de rédacteurs, d'un lieu comme étant touristique. Ce matériau implique donc une vision spécifique des processus de mondialisation du tourisme, qui est celle constituée à travers les guides touristiques.

\section{Déterminer la touristicité changeante des lieux}

Un deuxième problème se pose : la fonction touristique d'un lieu n'est pas éternelle et les trajectoires de "sortie" du tourisme sont légion (Équipe Mit, 20। I). Il convient donc de produire des données non seulement sur l'émergence des lieux, mais aussi sur la manière dont ils évoluent. C'est ainsi que l'on pourrait apprécier, avec davantage de précision, l'évolution quantitative des lieux touristiques, en prenant en compte non seulement la production de lieux touristiques, qui se renouvellent sans cesse depuis le début du XVIII siècle, mais aussi la reproduction et la fin 
Figure 1 • Parcours des lieuX touristiques

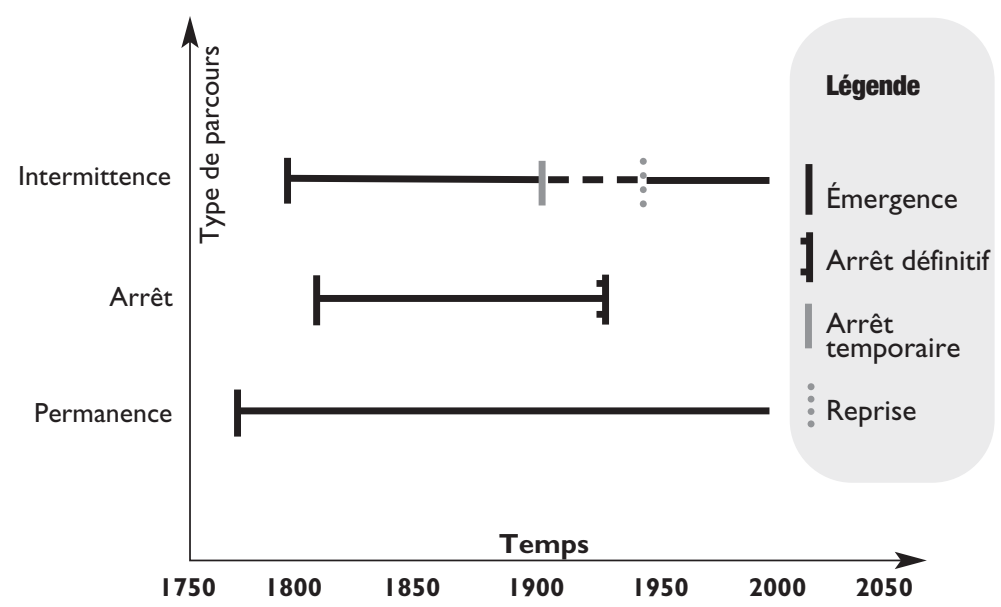

de la touristicité des lieux. C'est pourquoi il nous faut également déterminer les lieux qui ont cessé d'avoir une fonction touristique et le moment d'arrêt de cette fonction, que cet arrêt soit temporaire ou définitif. On distingue ainsi trois situations différentes (cf. figure 1) : l'arrêt touristique, qui se traduit par l'absence définitive de la fonction touristique d'un lieu ; l'intermittence qui se traduit par un arrêt temporaire, suivi par une reprise, de la fonction touristique d'un lieu au cours de son évolution ; la permanence touristique, qui est d'un lieu gardant sa fonction touristique tout le long de son existence.

\section{LE PASSAGE}

PAR LES GUIDES TOURISTIQUES

Le guide touristique (imprimé ou numérisé) est un moyen d'information touristique utilisé par le touriste.
C'est un instrument textuel qui sert d'introduction à la découverte d'un espace inconnu ou insuffisamment connu, à la promotion des sites et à l'imaginaire des touristes. Il peut contenir des informations pratiques (par exemple, où se loger, où manger, quels lieux visiter), des informations géographiques (descriptions de paysages, informations d'orientation dans le territoire, etc.), mais aussi des informations d'ordre culturel, historique, politique... Il offre une lecture, parmi d'autres, de l'espace touristique, et trouve sa spécificité dans la préférence accordée à certains lieux, jugés dignes d'intérêt, par rapport à d'autres, non mentionnés. Ainsi, il opère une sélection et une distinction sur un critère, le "regard touristique" du moment "médié" par les intérêts de la maison d'édition $^{(3)}$. Les adjectifs utilisés pour justifier cette sélection - "pittoresque", "paradisiaque", "exceptionnel", "extraordinaire"... - sont autant d'éléments intervenant dans la définition du touristique. En même temps, le genre "guides de voyage" est très vaste et diversifié : de multiples types coexistent et émergent au cours du temps, entre guides généraux, guides locaux et guides thématiques ${ }^{(4)}$. Les choix méthodologiques sur les lieux touristiques ont été opérés à partir des guides généraux.

\section{Apports \\ de cette source documentaire}

Le guide exprime le "collective tourist gaze” (Urry, 1990, p. 150). En effet, le guide touristique ne prescrit que les lieux touristiques qui jouissent, à un moment donné, d'une reconnaissance de leur statut touristique par différents acteurs des configurations sociales. L'analyse du guide touristique permet donc d'historiciser le tourisme et de reconstruire la façon dont l'espace touristique a été imaginé à un moment donné. Il s'agit de jugements sur des lieux géographiques qui sont contingents mais non arbitraires, et changeants au fil du temps.

L'une des raisons d'utiliser le guide touristique comme source pour l'analyse géo-historique du tourisme réside dans la datation des éditions. Tout d'abord, on peut dater les éditions, depuis les premiers guides autour de 1800 jusqu'à aujourd'hui. L'une des conditions pour cette approche géo-historique du tourisme est donc réunie. De plus, à intervalles réguliers, les guides d'une même maison d'édition sont réédités, ce qui permet la 
comparaison entre plusieurs éditions ; le chercheur peut en déduire la datation des lieux touristiques. Qui plus est, il existe une multitude de guides qui, portant sur les mêmes découpages géographiques, n'évoquent pas nécessairement les mêmes lieux touristiques. En cumulant les guides et les éditions, un grand nombre d'informations pourrait ainsi être extrait. De surcroît, l'une des entrées privilégiées des guides concerne les lieux que le touriste est censé "pratiquer”, de quelque manière que ce soit. Le guide centre donc son attention sur l'un des éléments majeurs de notre étude : les lieux touristiques. Enfin, l'accessibilité à la source et la standardisation de l'information contenue dans les guides sont des critères importants. Même si l'accès aux guides est loin d'être facile ${ }^{(5)}$, une certaine quantité et qualité d'informations sont assurées. Par contre, une source qui fonctionne sur le principe "demande-réponse" peut fournir un corpus d'informations incohérent, restreint ou focalisé sur un espace ou une période de temps ${ }^{(6)}$.

Les guides touristiques remplissent donc plusieurs conditions qui permettent de construire une connaissance de l'écoumène touristique et deviennent ainsi source d'investigation scientifique. Ils peuvent fournir des informations exploitables pour déterminer la date de mise en tourisme, l'arrêt définitif ou temporaire, ou encore la continuité de la fonction touristique d'un lieu. C'est une source qui répond à nos besoins en termes d'informations, sur le plan tant temporel que spatial.

\section{Limites}

\section{de la source documentaire}

On peut néanmoins pointer plusieurs limites de ce corpus. D’abord, il s'agit d'un corpus textuel soumis à un ensemble de règles éditoriales dont il faut être conscient. Il s'agit d'un certain point de vue sur l'espace touristique. Pour le scientifique, cela signifie que l'interprétation ne peut être celle de la mondialisation $d u$ tourisme, mais celle de la mondialisation d'un des aspects du tourisme qui donne accès à la constitution progressive de l'une des dimensions de l'espace touristique. Cette dimension relationnelle et relative doit être prise en compte, sous peine d'erreurs interprétatives. Notamment, le mode “découverte" est privilégié par les guides, bien que des guides thématiques existent, par exemple sur les villes d'hiver de la Méditerranée, les stations de ski ou encore sur les stations thermales ${ }^{(7)}$.

Ensuite, l'approximation des dates par les guides ne permet pas de répondre à la question de la datation absolue des événements historiques, en l'espèce l'émergence des lieux touristiques. La date retenue constitue donc une construction dont il convient d'apprécier les limites. Elle ne correspond pas à la première manifestation de l'événement, mais à son occurrence dans un corpus textuel. Cela a des conséquences sur les cas singuliers qui ne correspondent pas à la datation obtenue par le corpus de guides. Les datations singulières pourraient être reconstruites par un travail scientifique, au cas par cas.
Enfin, l'accès aux guides pose problème. Où trouver les collections suffisamment complètes de guides ? Nous avons exploré deux voies. Tout d'abord, celle des guides numérisés, en libre accès sur internet, et nous avons constaté que la recherche, à partir des mots-clés, n'est pas très aisée $^{(8)}$. En effet, les différentes platesformes en ligne n'utilisent pas les mêmes règles pour nommer un guide. Ainsi, les titres sont souvent incomplets, il y a une confusion ou un mélange entre les auteurs, les éditeurs et la collection de guides ${ }^{(9)}$. Nous nous sommes ensuite tournés vers les guides livres (papier), et le constat a été similaire : l'accès aux archives et bibliothèques a été problématique $^{(10)}$; les collections ne sont pas complètes, même dans les bibliothèques et archives spécialisées ${ }^{(11)}$.

Néanmoins, malgré ces contraintes, le corpus des guides est un matériau intéressant, à la fois en termes de lieux sélectionnés et de qualité décrite. Le moment de naissance du genre littéraire, la variété des entités spatiales couvertes et la densité des éditions sont les raisons pour lesquelles nous avons fait le choix de travailler à partir des guides de voyage.

\section{LA CONSTITUTION DU CORPUS TEXTUEL}

Une fois ce choix des guides touristiques arrêté, il faut s'intéresser à la façon dont on les sélectionne. Une analyse du genre littéraire nous a conduits à des sélections sur la base desquelles nous avons pu construire des bases de données sur les lieux 
touristiques à l'échelle mondiale. Cette analyse nous a permis de remarquer plusieurs choses. Premièrement, les guides sont organisés par collections qui prennent souvent le nom du fondateur ou de l'éditeur principal (les guides Joanne, Michelin, Murray, Baedeker, etc.) ; ils peuvent aussi prendre un nom qui renvoie à la couleur de la couverture du guide (les Guides Bleus, le Guide Vert) ${ }^{(12)}$. Deuxièmement, chaque collection produit des séries de guides (ou titres) décrivant des entités spatiales (South American Handbook, Guide to South and East Africa...). Comme toute publication littéraire, les titres des guides sont très divers et portent en général sur les entités géographiques décrites. Chaque série de guide (ou titre) comporte un nombre d'éditions qui peut varier en fonction de la politique éditoriale, de la demande et de la durée de publication de la collection. Troisièmement, le nombre des éditions peut renseigner sur la longévité de la collection. Des collections ont une période de publication impressionnante, comme les guides Baedeker (depuis 1828), les guides Murray (1836-1970) ou les guides appartenant à la maison Hachette (les Guides Joanne de 1841 à 1919, les Guides Bleus depuis 1919); à l'inverse, certaines collections n'ont pas réussi à s'imposer sur le marché, tels les guides Richard (1823-1836) ou les guides Napoléon Chaix (18481864). Quatrièmement, en fonction de l'origine éditoriale, chaque collection reflète un style et une vision spécifiques. Malgré ces différences, un "regard touristique commun" est appliqué au monde, un monde dans lequel les différences entre guides s'effacent au profit d'une vision semblable des lieux géographiques.

La prise en compte de ces éléments est cruciale lors de la constitution du corpus à partir des guides. En outre, afin de disposer d'un corpus cohérent et homogène, une coordination dans le temps et dans l'espace des différentes collections est nécessaire.

\section{Collections, séries, éditions : lesquelles choisir ?}

Plusieurs éléments contextuels sont importants à prendre en compte : un guide est publié par un éditeur ou une maison d'édition, il est réédité à certains intervalles, il est consacré à un espace géographique singulier et il peut être publié en plusieurs langues, avec des variantes.

- En ce qui concerne le choix des éditeurs, nous avons décidé de ne retenir que les grandes collections de guides : Baedeker, Murray, Guides Joanne, Guides Bleus, Guide du routard, Lonely Planet, etc., tout en vérifiant qu'ils couvrent tous les continents ( $c f$. figure 2). Toutefois, afin de disposer de données avant l'apparition des grandes collections (à partir de 1830), nous avons sélectionné parmi les auteurs les plus connus et les plus publiés de l'époque, par exemple Reichard et son successeur Richard.

Les différentes maisons d'édition ne proposent pas le même degré de précision et, partant, le même nombre de lieux touristiques. En termes de quantité, il y a de grands écarts. Par exemple, le Guide Delta sur l'Italie de 1982 propose 400 lieux, alors que les Guides Bleus pour le même pays proposent, en 1988, 1693 lieux. C'est pourquoi notre choix concernant les grandes collections se justifie, car le but est d'avoir une image détaillée des lieux touristiques dans le monde. De plus, pour disposer d'un corpus cohérent il est nécessaire que les différentes collections de guides soient comparables en termes d'espaces décrits, de périodes de publication et d'organisation générale du texte.

- Une seconde opération, très importante car elle constituera la base sur laquelle vont s'effectuer les analyses finales et la cartographie, est la sélection en fonction du "référentiel géographique" ${ }^{(13)}$ du guide. Pour pouvoir collecter un maximum de lieux à partir d'un guide de voyage, nous avons retenu les guides qui proposent un nombre important de lieux. Nous avons donc éliminé tout guide local (qui décrit, en général, un seul lieu) ou régional (y compris les guides portant uniquement sur les départements ou sur d'autres unités administratives inférieures à l'échelle nationale). Nous avons privilégié les espaces nationaux et les regroupements d'espaces nationaux et régionaux. Il faut noter que, très souvent, les éditeurs mentionnent le référentiel géographique dès le titre du guide. Ainsi, nous avons choisi des titres couvrant une superficie territoriale importante ${ }^{(14)}$. 


\section{RECHERCHE • VARIA}

Figure 2 • Sélection en fonction de l'Éditeur

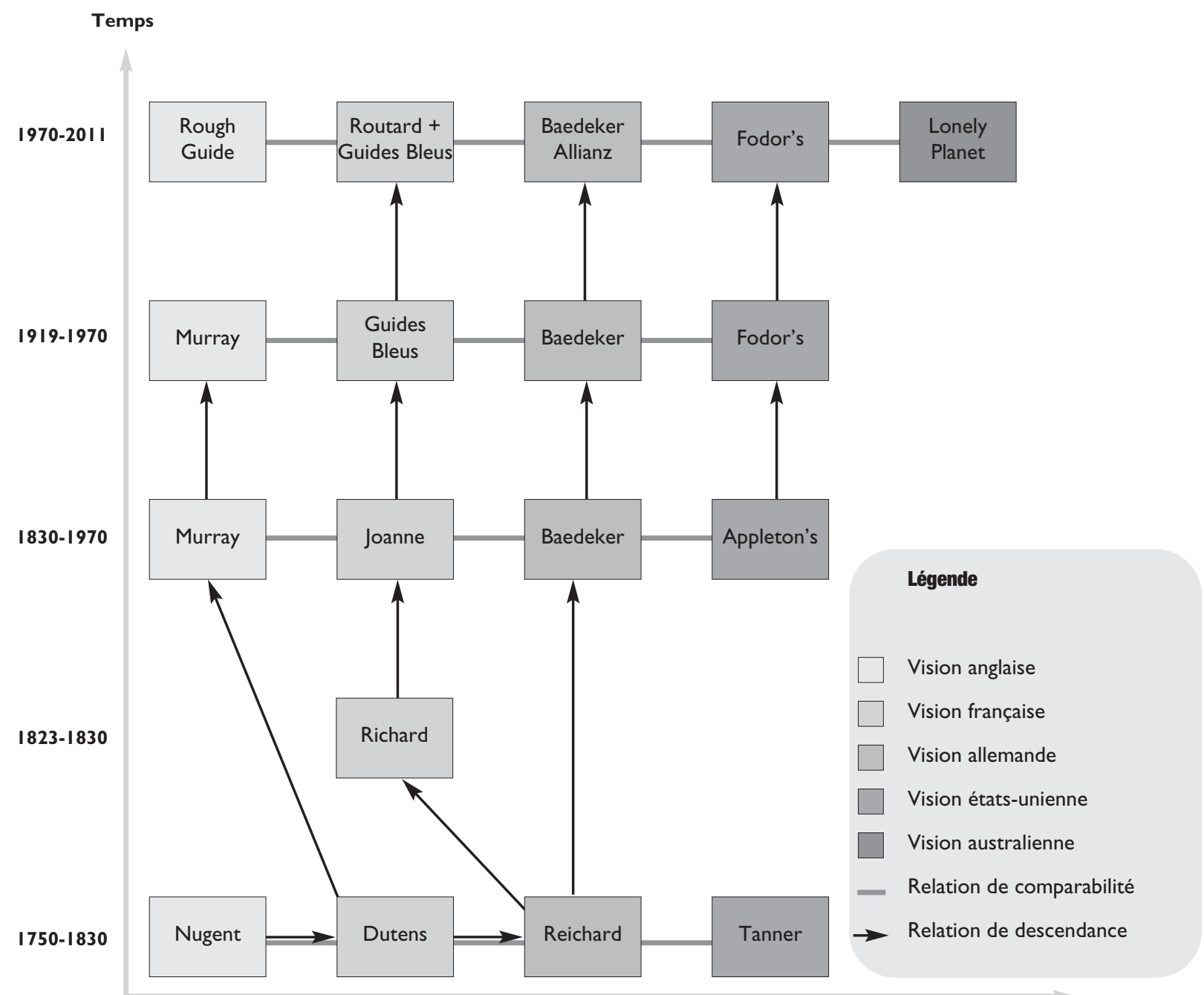

- La troisième opération est celle de la sélection en fonction de la mise à jour des éditions. Pour bien correspondre aux besoins des touristes et pour suivre les changements du moment, l'information d'un guide de voyage est renouvelée fréquemment ou actualisée. Dès le XIX ${ }^{e}$ siècle, il y a des mises à jour annuelles ou bisannuelles. Ces mises à jour peuvent comprendre des cor- rections d'erreurs faites dans les éditions antérieures, des informations supplémentaires sur un aspect du voyage (vie politique, précisions de distances, nouveaux éléments pratiques, nouveaux lieux touristiques, etc.). Notre intérêt se portera uniquement sur l'augmentation ou la diminution du nombre de lieux touristiques proposés. La méthode consiste à noter tous les lieux men- tionnés dans un guide touristique, tout en vérifiant s'ils ont fait l'objet d'une mise à jour dans les éditions suivantes ou antérieures, selon le cas. Une connaissance approfondie de l'évolution temporelle de chaque collection choisie est donc nécessaire. Les sélections en fonction de la mise à jour s'appliquent entre la première et la dernière édition d'un titre (cf. figure 3 ). 
Figure 3 • Périodes de publication des différentes collections de guides

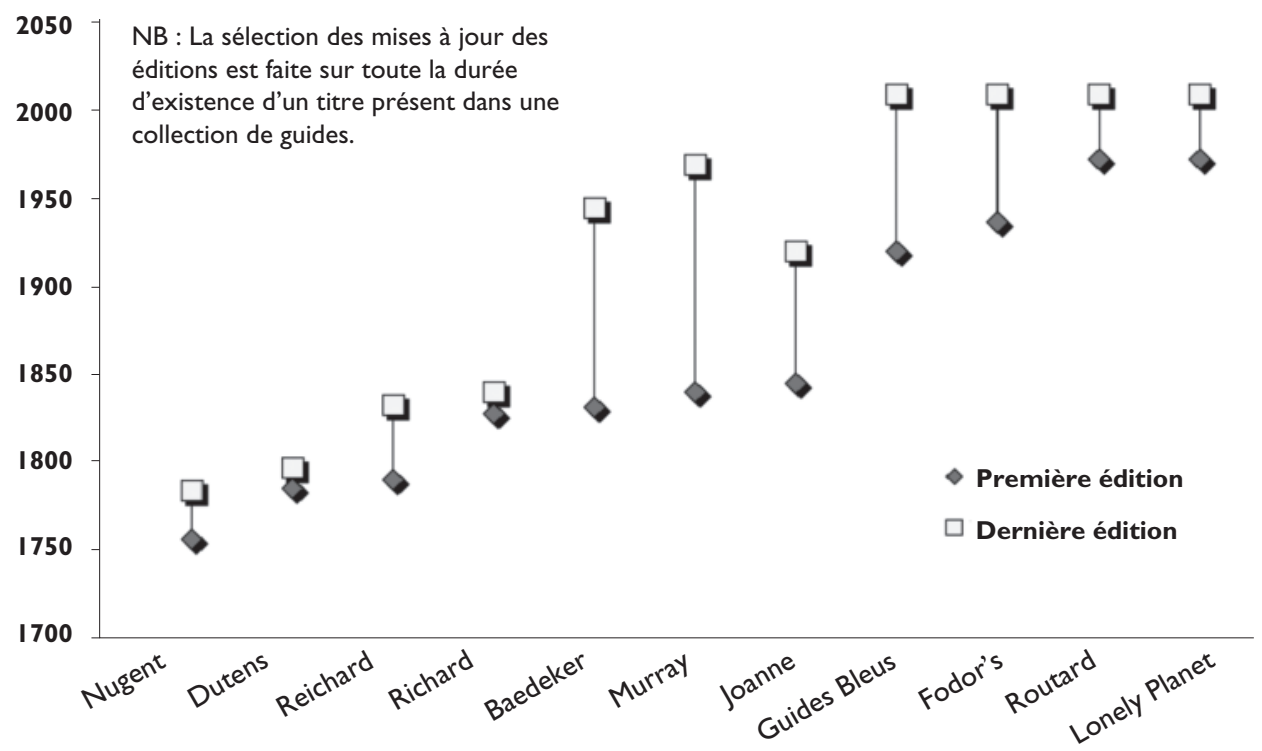

\section{Limites du corpus}

Le corpus ainsi constitué présente plusieurs limites. D'abord, il s'agit d'une vision certes dominante mais restreinte sur le monde touristique, car les seuls guides considérés sont les guides allemands, français, britanniques et états-uniens. Ce regard occidental exclut du champ du tourisme un certain nombre de lieux. Les lieux autres que ceux fréquenté par les Occidentaux n'apparaissent pas dans les guides ${ }^{(15)}$. Ensuite, il n'y a pas d'informations avant l'apparition des premiers guides, vers 1780. Les lieux émergeant avant cette date ne peuvent donc être traités de façon différenciée : ils apparaissent comme touristiques seulement à cette date. Cela correspond au consensus actuel sur l'histoire du tourisme : est consi- dérée comme "touristique" la période post-1800 ; est considérée comme "proto-touristique" la période entre 1700 et 1800 , laquelle concerne notamment les stations thermales (Tissot, 2000 ; Équipe Mit, 2005, 20 I I).

Enfin, les espaces décrits par les guides ne sont pas toujours comparables, certains d'entre eux ayant émergé touristiquement plus tôt que d'autres. Ainsi, certaines aires ne disposent pas ou presque pas de guides touristiques à une certaine époque et les mises à jour se font peu fréquemment. Tout dépend de la date d'émergence et de l'importance touristique de chaque aire géographique. Il y a donc une inégalité de traitement de la part des maisons d'édition qui influe sur les données constituées.

\section{L'EXTRACTION DES DONNÉES ET LA CONSTRUCTION DES BASES DE DONNÉES}

Une fois le corpus textuel sélectionné, l'étape suivante consiste à constituer le matériau empirique, à extraire les données et à construire une base de données qui comporte les dates d'émergence des lieux touristiques. On procède en deux étapes, la première étant consacrée à la constitution d'une base de données par guide qui comporte les différents toponymes issus de l'index alphabétique à une date donnée. La seconde étape concerne la constitution d'une base de données intégrant les différentes bases de données en une seule, constituée des différents toponymes extraits de tous les guides. On dispose ainsi des informations sur un toponyme à toutes les dates où il est mentionné entre 1800 et 2000. 


\section{L'extraction de l'information guide par guide}

Afin d'extraire les informations nécessaires à partir des guides choisis, nous considérons les guides comme des listes de lieux touristiques ${ }^{(16)}$ dont il faut identifier le toponyme et la date d'édition. Nous commençons par dépouiller les premiers guides apparus et suivons les différentes éditions publiées. Les lieux mentionnés dans les premiers guides sont considérés comme étant les premiers lieux touristiques ; la date de leur mise en tourisme retenue correspond à la date de la première édition qui les cite. Dans les éditions ultérieures, les nouveaux lieux touristiques mentionnés sont extraits avec leur date d'édition. Lorsqu'un lieu touristique signalé dans les éditions précédentes n'apparaît plus, on peut en déduire un arrêt de sa touristicité, les éditeurs ne considérant plus ce lieu comme touristique.

Se pose ensuite la question de l'extraction des lieux touristiques. Lors des analyses tests effectuées sur le corpus de guides, nous avons remarqué que les lieux mentionnés dans le texte figuraient aussi dans l'index alphabétique ${ }^{(17)}$, localisé en général à la fin du guide. Pour des raisons pratiques évidentes, nous avons proposé l'utilisation de cet index alphabétique comme plate-forme d'extraction des lieux touristiques. Cette opération d'extraction ou de saisie des lieux représente une phase de travail importante en termes de temps et de quantité d'informations. Elle est conduite en utilisant deux méthodes complémentaires : la saisie manuelle et celle assistée par un logiciel spécialisé dans la reconnaissance de texte ${ }^{(18)}$. Les lieux mentionnés dans l'index alphabétique sont ensuite versés dans une base de données ${ }^{(19)}$.

\section{Constitution d'une base de données comparative}

Une fois la collecte et la saisie des données finies, on passe à la constitution d'une base de données. Pour obtenir le nouvel ensemble de données concernant les différentes dates importantes de l'évolution des lieux touristiques, des opérations de comparaison sont nécessaires. Nous comparons les lieux mentionnés par les guides à des moments différents. Les moments choisis sont donnés par les éditions des guides. On crée ainsi un tableau à l'intérieur duquel on dispose, dans chaque colonne, l'année d'édition choisie pour l'espace en question. Un deuxième tableau est ainsi créé, représentant les résultats du traitement comparatif des deux éditions choisies (cf. figure 4).

Par exemple, pour les guides sur la Suisse, nous avons choisi de comparer les lieux mentionnés par les guides Murray de 1838 et de 1871. Pour déterminer les lieux qui deviennent touristiques (donc des émergences touristiques), on notera les lieux qui figurent dans l'édition de 1871, mais ne figurant pas dans la précédente, celle de 1838. Pour les lieux qui cessent d'être touristiques, on effectue la même opération, sauf que cette fois-ci on note les lieux qui apparaissent en 1838, mais ne figurent plus dans l'édition de 1871.
Pour les lieux touristiques permanents, le toponyme doit figurer dans les deux éditions. Ces opérations sont conduites à l'aide de formules conditionnelles qui cherchent chaque toponyme et l'affichent dans la colonne correspondante selon la commande donnée, et sont répétées jusqu'à la dernière édition de chaque titre de guide. Cela signifie que, pour pouvoir attribuer à un lieu l'une des trois qualités recherchées (émergence, permanence, arrêt touristique), on doit vérifier son existence (ou absence) dans toutes les éditions choisies.

Cette méthode implique que la date de mise en tourisme d'un lieu corresponde à la période précédant sa première mention dans un guide de voyage ${ }^{(21)}$. Par exemple, dans la figure 4, le lieu "Baar" apparaît pour la première fois dans l'édition de 1871 du guide Murray. Mais comme l'exhaustivité d'un suivi des éditions des guides n'a pu être envisagée dans le cadre de cette recherche et que la période entre les deux éditions comparées (ici 1838 et 1871) est relativement courte, nous considérons que la datation de l'émergence touristique de Baar et des autres lieux inscrits dans la même colonne devrait s'exprimer sous forme de période, et non sous celle de date fixe. Nous allons donc considérer que l'émergence touristique de Baar est intervenue dans la période allant de 1839 à 1871 , puisque l'édition de 1838 ne mentionne pas ce lieu. Ensuite, la même logique s'applique en ce qui concerne la date de l'arrêt touristique. On remarque l'absence de 


\section{Figure 4 • Exemple de traitement entre deuX Éditions de guides SUR La SuISSe (EXTRAIt PARTIEL dU TABLEAU D'ORIGINE)}

\begin{tabular}{|c|c|c|c|c|}
\hline $\begin{array}{c}\text { Edition de } \\
1838 \text { du Guide } \\
\text { Murray }\end{array}$ & $\begin{array}{c}\text { Edition de } 1871 \\
\text { du Guide } \\
\text { Murray }\end{array}$ & $\begin{array}{l}\text { Emergence } \\
\text { touristique entre } \\
1839 \text { et } 1871\end{array}$ & $\begin{array}{c}\text { Arrêt touristique } \\
\text { entre } \\
1839 \text { et } 1871\end{array}$ & $\begin{array}{l}\text { Permanence } \\
\text { touristique }\end{array}$ \\
\hline & Baar & Baar & & \\
\hline \multirow[t]{2}{*}{ Bachalp } & & & Bachalp & \\
\hline & Bachtel & Bachtel & & \\
\hline Baden & Baden & & & Baden \\
\hline \multirow[t]{3}{*}{ Bagnes } & Bagnes & & & Bagnes \\
\hline & Balbianello, Villa & Balbianello, Villa & & \\
\hline & Baldegg & Baldegg & & \\
\hline \multirow[t]{4}{*}{ Bâle } & Bâle & & & Bâle \\
\hline & Balen & Balen & & \\
\hline & Balferinhorn & Balferinhorn & & \\
\hline & Balliswyl & Balliswyl & & \\
\hline \multicolumn{5}{|l|}{ Ballsthal } \\
\hline \multirow[t]{3}{*}{ Balme } & Balme & & & Balme \\
\hline & Balmhorn & Balmhorn & & \\
\hline & Balmuccia & Balmuccia & & \\
\hline \multirow[t]{4}{*}{ Balsille } & Balsille & & & Balsille \\
\hline & Balzers & Balzers & & \\
\hline & Bannio & Bannio & & \\
\hline & Barberine & Barberine & & \\
\hline Barcelonette & Barcelonette & & & Barcelonette \\
\hline Bard & Bard & & & Bard \\
\hline
\end{tabular}

NB : Les tableaux d'origine, organisés alphabétiquement, contiennent tous les lieux mentionnés par chaque édition. II s'agit ici d'un extrait visant à montrer le fonctionnement des formules utilisées pour le traitement des données.

formalisation de l'“arrêt touristique". Étant donné que l'on compare deux éditions du XIX siècle et que l'on ne connaît pas encore le contenu des éditions suivantes (au moment de la comparaison entre seulement ces deux éditions), on ne sait pas s'il s'agit d'un arrêt temporaire ou définitif de la fonction touristique. C'est pourquoi, une vérification des lieux jusqu'à la dernière édition (et donc jusqu'à nos jours) est impérative. C'est une condition sine qua non de la détermination des arrêts, des intermittences, mais aussi des permanences de la fonction touristique.
À la fin, nous obtenons pour chaque lieu des dates assez précises sur le moment d'émergence, les éventuelles périodes intermittentes et le moment d'arrêt de la fonction touristique (cf. figure 1). Nous pouvons, en même temps, identifier les lieux qui ont maintenu leur touristicité tout le long de la période considérée (1800-2000).

\section{RÉSULTATS PROVISOIRES}

L'échantillon issu de cette collecte de données comprend environ 120000 lieux touristiques. Il s'agit du nombre total de lieux saisis à partir des guides touristiques choisis. Ce qui implique, en fonction du nombre des éditions dépouillées pour un même espace, que des lieux identiques peuvent se trouver plusieurs fois dans la base de données pour une même période. Une fois les toponymes en double décomptés, l'échantillon se trouve ramené à environ 40000 lieux touristiques singuliers avec date d'émergence et, le cas échéant, d'arrêt.

Les résultats provisoires s'appuient sur ces 40000 lieux touristiques pour lesquels on propose une cartographie et un ordre de grandeur de la densification de l'écoumène 
Tableau 1 - Quantification périodique des Émergences et des arrêts temporaires oU définitifs

\begin{tabular}{lrrrrrr} 
Période & $\begin{array}{r}\text { Émergences } \\
\text { moyenne annuelle }\end{array}$ & $\begin{array}{r}\text { Production } \\
\text { moyens annuels }\end{array}$ & $\begin{array}{r}\text { Arrêts } \\
\text { par période }\end{array}$ & $\begin{array}{c}\text { Arrêts } \\
\text { cumulé }\end{array}$ \\
\hline I793-1830 & 2410 & 65 & 1100 & 30 & +1310 & 1310 \\
I83I-1850 & 2799 & 147 & 1294 & 68 & $+\mid 505$ & 2815 \\
I85I-188I & 6030 & 201 & 4184 & 139 & $+\mid 900$ & $466 \mid$ \\
I882-1895 & 4080 & 318 & 4252 & 327 & -200 & 4489 \\
I896-1928 & 4350 & 135 & 5110 & 159 & -900 & 3729 \\
I929-1973 & 6537 & 148 & 5626 & 127 & +900 & 4640 \\
I974-2000 & 13582 & 522 & - & - & - & 18222 \\
\hline
\end{tabular}

touristique. On constate que le tourisme a investi progressivement le monde entier, tout en se concentrant sur un nombre de lieux ( $c f$. figure 5).

Si l'on raisonne en fonction des bornes temporelles construites à partir des données (méthode dite des seuils naturels), on peut faire les constats suivants ( $c f$. tableau 1$)$ : en termes d'émergence, la période 17931830 correspond à un vingtième environ de la période 1974-2000 ; elle est celle qui enregistre la plus faible production moyenne annuelle de lieux touristiques (sept fois moins que la période 1974-2000). Une première poussée d'expansion du tourisme s'observe à partir de 1830 , suivie par une deuxième, à partir de 1851, avec une multiplication par deux des lieux touristiques émergents par rapport à la période précédente et une production moyenne annuelle en hausse : entre 1851 et 1881,200 lieux touristiques émergent en moyenne par an ; entre 1882 et 1895 , ils sont plus de 300 par an. Cela correspond, d'une part, à une poussée de mondialisation importante que la Première Guerre mon- diale arrête et, d'autre part, à une première phase de tourisme dit "industriel" (Tissot, 2000 ; Équipe Mit, 20I I), au sens d'une massification importante. Spatialement, ces périodes sont caractérisées par une expansion au-delà de l'Europe et des États-Unis.

Une grande quantité de lieux cessent d'être touristiques entre 1896 et 1929 et la production annuelle moyenne tombe au niveau des années 1830. Cet effondrement des lieux touristiques est suivi d'une phase de réémergence, avec des ordres de grandeur au niveau de ceux du milieu du XIX siècle. Le tourisme de masse, qui se manifeste, à partir des années 1950, par l'augmentation des taux de départ dans les pays les plus développés, s'affirme peu pour ce qui est du nombre de lieux touristiques - leur production annuelle moyenne reste inférieure à l'ordre de grandeur de celui du milieu du XIX ${ }^{e}$ siècle. Ce n'est qu'à partir des années 1970 que le système touristique est en expansion, tant du point de vue quantitatif que spatial, avec une progressive couverture mondiale ( $c f$. figure 6). Sur l'ensemble de la période considérée, on peut donc mettre en évidence deux phases d'expansion rapide du champ touristique mondial, caractérisées par des productions annuelles élevées de lieux touristiques : entre 1851 et 1895 ; et entre 1974 et 2000.

On peut donc construire une cartographie plus précise que celle issue des travaux antérieurs (Équipe Mit, 20I I), car, outre la production des lieux touristiques, les relais, intermittences et arrêts définitifs de la fonction touristique peuvent aussi être cartographiés. On dispose désormais d'un ordre de grandeur - relatif, car nous raisonnons à partir d'un échantillon non exhaustif - du nombre de lieux touristiques et de celui des lieux émergents à différents moments de l'histoire.

\section{Conclusion}

L'un des apports de ce travail consiste en une méthodologie permettant de reconstruire avec plus de précision, grâce à la production de nouvelles données, le système 
Figure 5 • La densité des LIeuX touRistiques entre 1793 et 2000

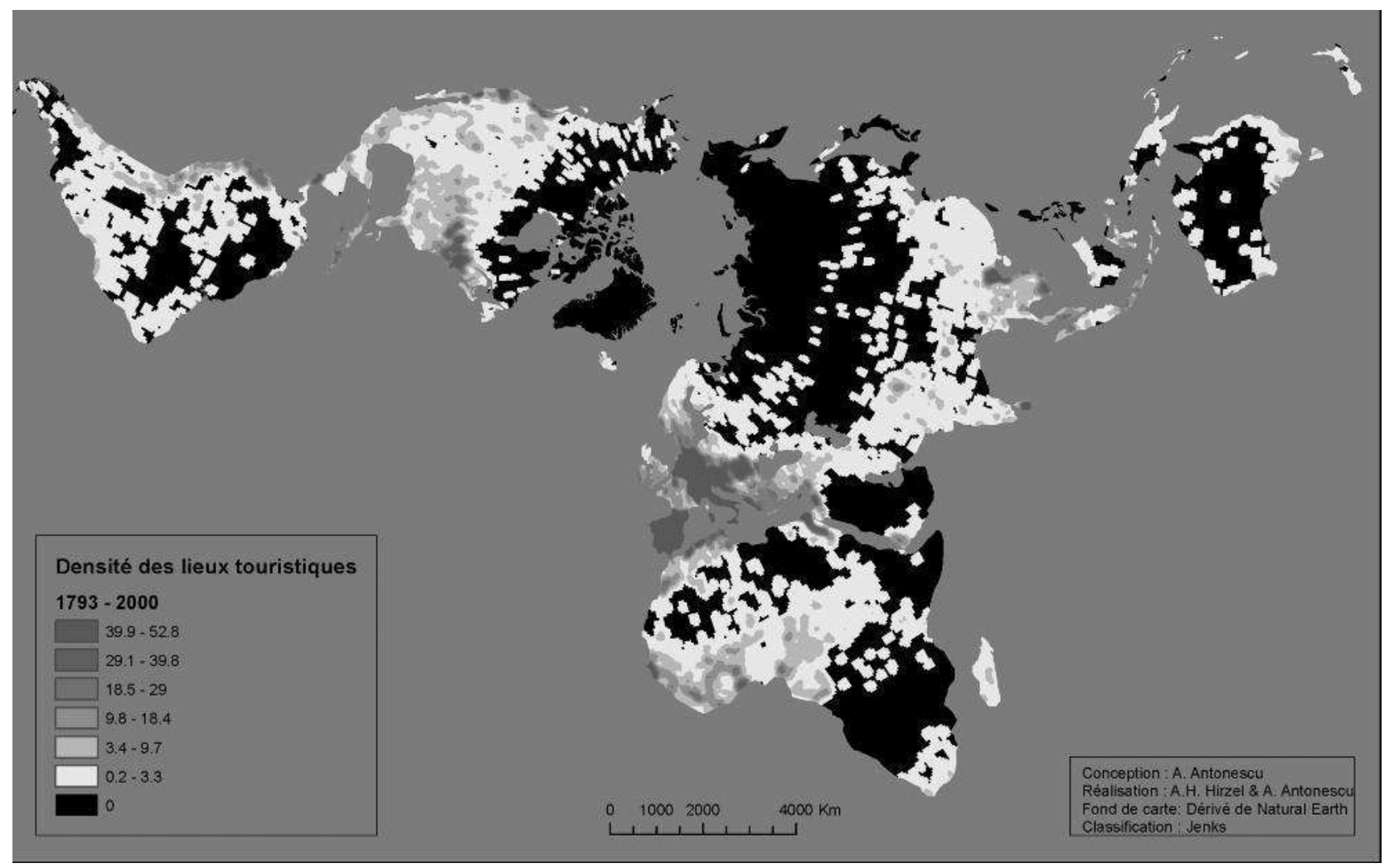

Figure 6 • Émergence de LIEUX TOURISTIQUeS À PLUSIEURS MOMENTS DE L'HISTOIRE

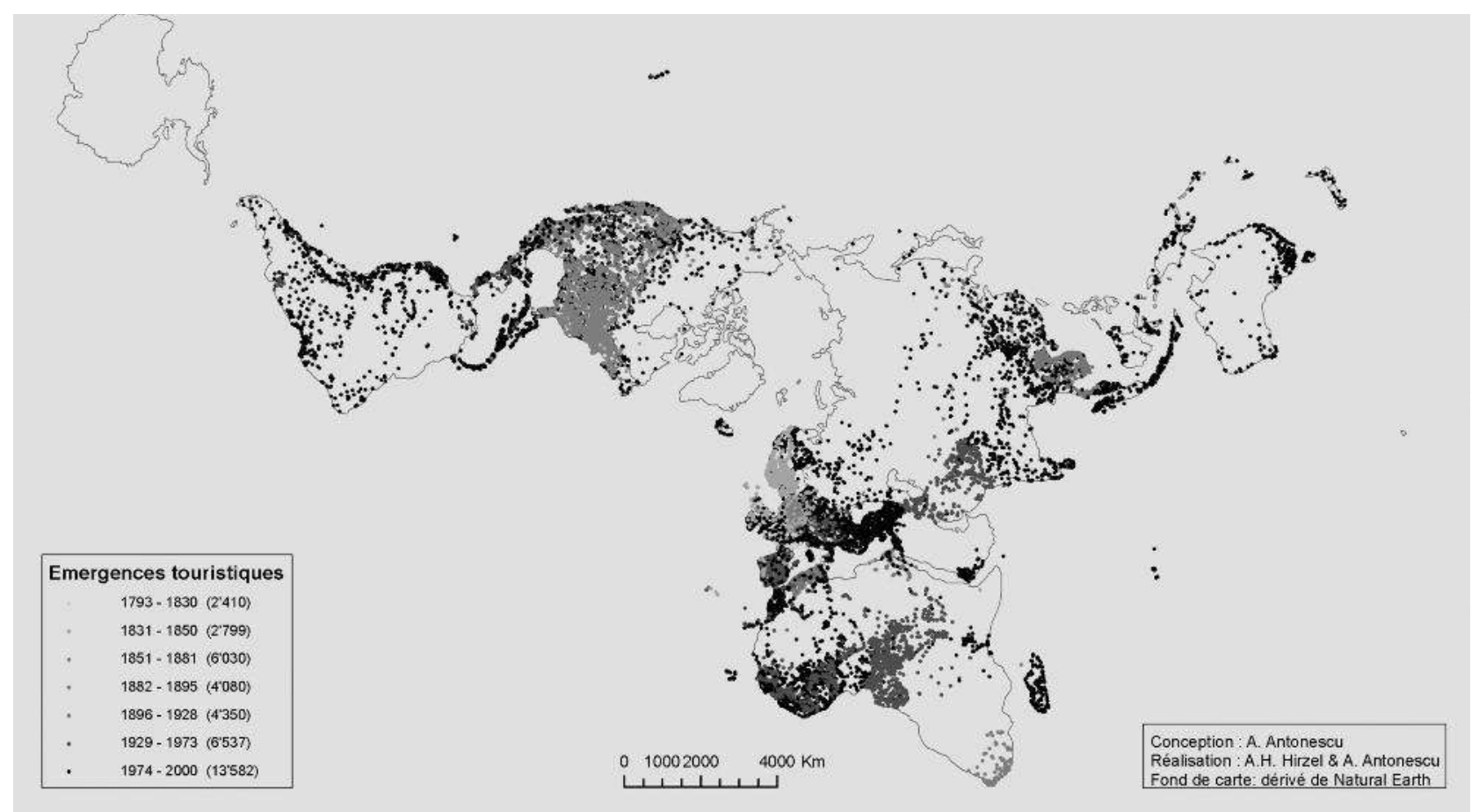


touristique changeant au cours du temps. Par son recours massif aux techniques de numérisation et de traitement par ordinateur, ce travail s'insère dans les "bumanités digitales”, bien que mobilisant des techniques classiques en géographie (comme celle du système d'information géographique).

Ce travail permet ainsi de quantifier le nombre de lieux touristiques nouvellement produits, le nombre de lieux dont la fonction touristique s'est arrêtée, et celui dont la fonction touristique s'est maintenue. Cette information quantitative est précieuse, car la recherche scientifique dispose maintenant d'une estimation des ordres de grandeur de l'émergence et de l'arrêt des lieux touristiques, ce qui va permettre de répondre à des questions qui ont interpellé, récemment, la communauté scientifique, comme, par exemple : combien de lieux touristiques ont-ils émergé entre 1800 et 1830 ? la production de lieux touristiques a-t-elle été plus forte entre 1830 et 1880 qu'entre 1929 et 1973 ? On peut ainsi donner des réponses en termes relatifs et comparer les moyennes annuelles, par période, de production et d'arrêt de lieux touristiques. La mondialisation du tourisme est ainsi davantage qualifiée : au lieu d'une histoire linéaire d'expansion mondiale à partir du foyer européen, on remarque des périodes d'accélération et d'arrêt qui correspondent partiellement aux phases de la mondialisation économique, notamment l'expansion du système touristique jusqu'à la
Première Guerre mondiale et l'émergence d'un système mondial du tourisme à partir des années 1970.

Il s'agit là d'un premier pas vers la conception et la vérification empirique d'un "champ touristique mondial” (Clivaz et al., 20। I) dans lequel les lieux touristiques seraient interdépendants. En effet, le champ touristique se caractérise, à différents moments de l'histoire, par son inégale ampleur et sa spatialité changeante. Ainsi, le champ du tourisme n'est pas mondial dans la première phase, mais se concentre sur l'Europe et les États-Unis au début du xixe siècle. On constate ensuite une extension du champ du tourisme, aux États-Unis et en Europe, d'une part, et calqué sur l'expansion coloniale des Européens, d'autre part. Le champ touristique est véritablement mondial depuis les années 1970, époque à laquelle on constate que le tourisme n'est plus pratiqué en quelques lieux dispersés, mais qu'il investit le monde entier.

L'étape suivante pourrait consister à observer les changements (ou le maintien) de la qualité des lieux touristiques au cours du temps et les moments où ces manifestations se produisent. Peut-on distinguer les lieux par leur type (par exemple, selon la typologie de l'Équipe Mit (2002) - en ville, station, comptoir ou site touristique) ou par leur pratique(s) touristique(s) dominante(s), et ce pour l'ensemble des 40000 lieux touristiques de notre base de données ? Pour mettre en œuvre ce type de recherche, il faudra faire un effort important pour qualifier davan- tage les différents lieux touristiques, pour ce qui est, par exemple, des données concernant le nombre de meurs habitants (avec les évolutions, entre 1800 et 2000) et celui de leurs touristes ou de leurs nuitées touristiques (avec les évolutions, entre 1800 et 2000). Ce nouveau chantier est maintenant devant nous.

\section{Notes}

(*) Le présent article est issu du projet de recherche financé par le Fonds national suisse de la recherche (FNS), $n^{\circ}$ I2704I, "Mondialisation et tourisme. Une approche géo-historique".

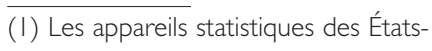
nation présentent différents degrés de précision et de profondeur historique. Par exemple, en Suisse, on dispose de données statistiques sur le tourisme à partir de 1937, en France à partir de 1949, dans certains Länder allemands à partir de 1925.

(2) Cf. la définition que donne l'Équipe Mit (2002, p. 300) de la mise en tourisme:

"Processus de création d'un lieu touristique ou de subversion d'un lieu ancien par le tourisme qui aboutit à un état : le lieu touristique." (3) Cf. Roland Barthes (1957) pour une critique de ce genre littéraire dont le regard sélectionne le "pittoresque" ; et cf. Hécate Vergopoulos (20II) pour un traitement de "l'insolite" comme élément structurant du regard du guide touristique.

(4) Cf. Pierre-Yves Saunier (1994), Gilles Chabaud et al. (2000), Goulven Guilcher (2005), Hélène Morlier (2007) pour cette littérature.

(5) Ce point sera fréquemment abordé dans les parties destinées aux limites méthodologiques.

(6) Nous avons effectué quelques tests auprès des offices de tourisme européens 
en demandant par e-mail des informations sur les lieux touristiques du pays interrogé. Le taux de réponses a été extrêmement faible et, parmi les réponses fournies, certaines étaient très disparates et non exploitables du fait de l'absence de données ou encore de l'incompréhension de la demande, même si nous avions utilisé des termes simples et clairs.

(7) Par exemple, les 17 éditions des

Guides Joanne consacrées aux Stations d'hiver de la Méditerranée entre 1875 et 1906, les guides Appleton sur les American Winter Resorts ou encore les guides de Edwin Lee sur les Watering Places and Mineral Springs.

(8) Pour les guides anciens édités jusqu'en 1910-1920, le problème des droits d'auteur ne se pose pas. Cf., par exemple, www.archive.org pour une collection de guides touristiques.

(9) II faut savoir qu'une maison d'édition de guides collabore avec plusieurs auteurs, souvent inconnus. Si l'on cherche un guide en fonction de la maison d'édition, il est très possible que le titre recherché ne soit pas trouvé, parce qu'il a été répertorié sous le nom de son auteur. Par exemple, pour les guides Appleton, en Amérique du Nord, nous avons trouvé des auteurs connus, comme Daniel Appleton ou William Wellington, mais nous avons aussi trouvé des guides écrits par Willis Pope Hazard ou le Colonel Fremont. Autre problème : ces guides ont été réimprimés ou réédités par d'autres auteurs dont on ne connait pas le nom. D'où une confusion entre le nom de la collection (qui est souvent le nom de la maison d'édition ou de l'éditeur chargé de la collection) et les auteurs de ces guides. Par exemple, les guides Reichard ont été publiés par différents éditeurs, tels que Herbig, Hyacinthe Langlois, Audin, Samuel Leigh, Gandini. Un autre exemple est celui des guides Richard qui ont été publiés par des éditeurs comme Louis Maison, Audin et Hachette. Hachette a d'ailleurs acheté certains titres écrits par des auteurs, en particulier ceux de Audin, Mariana Starke, Ebel, Lutz (Morlier, 2007).

(10) À part les formalités administratives et les demandes d'autorisations spéciales (pour consulter une certaine collection de livres et pour avoir le droit de photographier), les difficultés sont notamment les suivantes : un temps très limité sur place pour un corpus très large, une multitude de salles de lecture à fréquenter, l'utilisation du scanner à partager avec les autres lecteurs, des guides manquants, le temps long d'attente pour les demandes de guides.

( I I) La Bibliothèque nationale de France, la Library of Congress de Washington ou la National Library de Londres sont des ressources-clés. On peut également recourir aux archives de l'Abbaye d'Ardenne, près de Caen, (pour les guides Hachette) et aux archives sur le tourisme de l'université technique de Berlin (Historisches Archiv zum Tourismus).

(I2) Cf. exemples dans la partie bibliographique.

(I3) Le terme est utilisé ici dans le sens d'entité spatiale nommé par le guide touristique.

(14) Par exemple, des titres de guide portant sur des entités spatiales, comme Austria (Guide Baedeker, I 896), Belgique et Luxembourg (Guides Bleus, 1927), Pays nordiques (Guides Bleus, 1955), European Guide (Appleton's Guide, I887), ou encore Orient (Guides Joanne, I873).

(15) II faudrait, lors d'une recherche ultérieure, étendre le corpus aux collections japonaises, chinoises, canadiennes, brési- liennes, argentines, etc.

( 16) Ce choix est discutable, car on peut noter une évolution dans la manière dont les guides traitent les lieux touristiques, par rapport aux autres lieux qu'ils citent.

Toutefois, cela nous permet d'avoir une source cohérente sur ce qu'un guide considère comme lieu touristique à un moment donné.

(I7) L'index alphabétique est le répertoire de tous les lieux mentionnés dans le guide, mais il peut aussi contenir des noms de personnages de l'époque ou encore des mots-clés utilisés dans le texte.

(18) Les lieux mentionnés dans l'index alphabétique sont copiés soit manuellement soit à l'aide du logiciel ABBYY, spécialisé dans la reconnaissance optique des caractères (OCR). Ces deux méthodes de saisie des données sont très souvent complémentaires.

(19) Concrètement, nous avons utilisé le scan ou la photographie des pages des guides pour les transformer ensuite en fichier Excel. Ainsi, nous disposons, pour chaque guide, d'une première base de données, que nous avons appelée "base de données initiale". Toutes les bases de données initiales seront ensuite intégrées dans une seule base, nommée "base de données finale".

(2I) II s'agit d'une des limites posées par les guides touristiques : c'est le guide qui fait foi, pas la date à laquelle le premier touriste pratique un lieu. De nouveau, c'est la cohérence de la source qui préside à ce choix. 


\section{ÉCHANTILLON DU CORPUS DE GUIDES TOURISTIQUES}

- Appleton's Guides, American Summer Resorts, 1876.

- Appleton's Guides, American Winter Resorts, 1877.

- Appleton's, European Guide, 1887.

- Baedeker, Austria, 1896.

- Baedeker, Belgien und Holland, 1880.

- Baedeker, Deutschland, die Bundesrepublik. Offizieller Führer des Allgemeinen Deutschen Automobil-Clubs, 1953.

- Baedeker, Palestine and Syria, 1894.

- Bibliothèque des chemins de fer, De Lyon à • a Méditerranée, 1855.

- Bibliothèque des chemins de fer, De Paris à Bordeaux, 1856.

- Bibliothèque des chemins de fer, De Paris à Boulogne, à Calais et à Dunkerque, 1855.

- Bibliothèque des chemins de fer, De Paris au centre de la France, 1854.

- Edwin Lee, English Watering Places, 1849.

- Edwin Lee, The Health Resorts of the South of France, 1868.

- Edwin Lee, The Mineral Springs of England and their Curative Efficacy, 1841 .

- Fodor's Guides, Australia, New Zealand and South Pacific, 1978.

- Fodor's, New York City and State, 1979.

- Fodor's, San Francisco with Nearby Attractions, 1982.

- Fodor's Guides, National Parks and Seashores of the East, 1996.

- Fodor's Guides, Ski Resorts of North America, 1986.

- Fodor's Guides, The Complete Guide to the National Parks of the West, 2012.

- Frommer's Guide, Family Vacations in the National Parks, 2004.

- Guide Delta, Italie, 1982.

- Guide du routard, Amérique du Nord et centrale, 1979.

- Guide du routard, Bourgogne et Franche-Comté, 1998.

- Guides Joanne, Bains de mer de

Normandie, 1913.

- Guides Joanne, Europe, 1860.

- Guides Joanne, Orient

(Grèce et Turquie d'Europe), 1873.
- Guides Joanne, Orient (Malte, Égypte, Nubie, Abyssinie, Sinai), 1890.

- Guides Joanne, Stations d'hiver de la Méditerranée, 1875.

- Guides Bleus, Algérie et Tunisie, 1930.

- Guides Bleus, Belgique et Luxembourg, 1927.

- Guides Bleus, Bretagne, 1982.

- Guides Bleus, France automobile en un volume, 1954.

- Guides Bleus, Italie, 1988.

- Guides Bleus, Languedoc-Roussillon, 1968.

- Guides Bleus, Pays nordiques, 1955.

- Guides Reichard, Guides des voyageurs en Europe, 1793.

- Guides Reichard, Handbuch für Reisende durch Europa, 1784.

- Murray, Asia Minor, Transcaucasia and Persia, 1905.

- Murray, Devon and Cornwall, 1859.

- Murray, English Lakes, 1889.

- Murray, Essex, Suffolk, Norfolk and Cambridgeshire, 1875.

- Murray, London, 1851.

- Murray, Rome and its environs, 1867.

- Murray, Switzerland, 1838.

- Murray, Switzerland, 1871.

- Thomas More Madden, The Spas of Belgium, Germany, Switzerland, France and Italy, 1867. 


\section{Références bibliographiques}

Roland BARTHES, Mythologies, Seuil, 1957.

Gilles Chabaud, Évelyne Cohen, Natacha Coquery et Jérôme Penez (dir.), Les Guides imprimés du XVe au XXe siècle. Villes, paysages, voyages, Belin, 2000.

Christophe ClivaZ, Stéphane NAHRATH et Mathis STOCK, "Le développement des stations touristiques dans le champ touristique mondial", dans Philippe DUhamel et Boualem KaDRI (dir.), Mondialisation et tourisme, coll. "Mondes du tourisme", ETE, septembre 2011 .

Nicolas DupuY et Pascal MAO, Les Pratiques sportives de nature en Midi-Pyrénées. Atlas des sites de pratique, Éditions du CNRS, 2003.

Équipe MIT, Tourismes I. Lieux communs, Belin, 2002.

Équipe Mıт, Tourismes 2. Moments de lieux, Belin, 2005.

Équipe Mit, Tourismes 3. La révolution durable, Belin, 2011.

Goulven GUILCHER, "Entre continuité et innovation, les guides touristiques en langue française de 1900 à 1960", dans Martine BREUILlot et Thomas BeauflLS (dir.), Le Guide de voyage au fil du Rhin et ailleurs, Université de Strasbourg-II, 2005.

Rémy KNafou, Atlas de France, vol. 7, Tourisme et loisirs, Gip Reclus/La Documentation française, 1997.

François MORICONI-EBRARD, L'Urbanisation du monde depuis 1950, Anthropos, 1993.

Hélène Morlier, Les Guides Joanne : genèse des Guides Bleus. Itinéraire bibliographique, historique et descriptif de la collection de guides de voyage, 1840-1920, Les Sentiers débattus, 2007

Guillaume OluIVIer, Ghislain GeniauX, Claude NAPOlÉONe et Jean-Christophe PAOLI, "Mesurer la notoriété touristique communale : contribution méthodologique à l'aide de l'analyse textuelle de guides touristiques", Cybergeo, n593, 2012

[http://cybergeo.revues.org/25|87].

Pierre-Yves SAUNIER, "Le guide touristique, un outil pour une possible histoire de l'espace : autour des guides de Lyon I800-19|4", Géographie et cultures, n¹3, 1994.

Laurent TIssot, Naissance d'une industrie touristique : les Anglais et la Suisse au XIXe siècle, Payot, 2000.

Richard S. J. Tol, Andrea Bigano, Jacqueline M. Hamilton, Maren LAU et Yuan ZHOU, "A global database of domestic and international tourist numbers at national and subnational level", International Journal of Tourism Research, vol. 9, n 3, 2007.

Céline TRITZ, Tourisme et espace : l'arc méditerranéen. Une approche de la médiatisation, thèse de géographie, Université de Nice-

Sophia Antipolis, 2003.

Céline TRITZ, "La valorisation touristique d'un territoire : entre acteurs et territoire. Proposition de méthodes et applications", communication au colloque international Développement territorial et tourisme durable, une approche par la valorisation touristique, Bogota, 2006

John URRY, The Tourist Gaze, Sage Publications, 1990

Hécate Vergopoulos, “L'insolite dans les guides touristiques. Voyage en 'Pataxie”', Mondes du tourisme, nº 4, décembre 201 I. 